\title{
CRISPR: Challenges to South African biotechnology law
}

\author{
S Pillay, BSc Hons, LLM; D W Thaldar, BLC, LLB, MPPS, PhD, PGDip (Strategy \& Innovation) \\ School of Law, Howard College, University of KwaZulu-Natal, Durban, South Africa
}

Corresponding author:DWThaldar (ThaldarD@ukzn.ac.za)

\begin{abstract}
Genome editing using newly discovered tools such as CRISPR-Cas9 offers the potential to modify the genetic make-up of plants, non-human animals and humans in exact ways. Such genetic modification can serve the purpose of correcting gene defects, and can enhance certain characteristics. Apart from reigniting old ethical debates, genome editing also poses a particular challenge to legal practitioners, as this new technology does not always fit into existing definitional moulds, and it lacks clear legal precedent. In this article, we identify the most salient areas of concern in the South African legal context.
\end{abstract}

S Afr J Bioethics Law 2018;11(2):89-92. DOI:10.7196/SAJBL.2018.v1 1i2.653

Gene editing is a technology that enables changes to an organism's DNA by adding, removing or altering genes so as to change gene expression. However, unlike genetic modification, which introduces foreign DNA (transgenes) into an organism, gene editing does not introduce transgenes - instead, the technology is limited to the DNA naturally present in the organism's gene pool (this point will be clarified further below). The new 'tool' that has proved highly successful and reliable to accomplish gene editing is CRISPR-Cas9. The discovery of CRISPR-Cas9 in bacteria, and its novel application in plant and animal cells, has been hailed as revolutionary. ${ }^{[1]}$ While CRISPR-Cas9 is being explored, analogous systems are also being discovered - such as CRISPR-Cas12a. ${ }^{[2]}$ These genome-editing tools are showing potential not just to correct gene defects, but also to enhance the phenotype. However, given the expanding possibilities offered by this technology, the legal, ethical and social issues are also becoming clearer and more pressing. ${ }^{[1]}$

Currently, there is international debate on how CRISPR-Cas9 should be regulated in applicable industries. Actors involved recognise the need to advance this technology to maximise benefits to society, but also the need to apply caution so as to protect public and environmental health. ${ }^{[3]}$ Central to these debates is the fact that CRISPR-Cas9 is a relatively nascent technology, the potential of which to impact on human, animal and environmental health cannot be determined with perfect clarity. This echoes the sentiments in the debate on genetically modified organisms (GMOs). However, CRISPRCas9 is not limited to agricultural biotechnology; it also extends to other areas, such as human biotechnology. In this latter context, the discovery of CRISPR-Cas9 has reignited the global ethical debate on human genetic engineering.

In this article, we provide an overview of CRISPR-Cas9 technology, and present key areas of concern in the South African (SA) legal context - pertaining to agriculture, human gene therapy, patenting and consumer protection. Our intention is to draw attention to potential ethical and legal problems in order to stimulate academic debate - not to suggest substantive solutions.

\section{The CRISPR revolution}

The manipulation of the DNA in genes has been possible for decades. Technologies have been invented since the DNA helical structure was first understood, but site-specific modifications in genomes were still unachievable. ${ }^{[4]}$ In recent years, site-specific technologies such as zinc finger nucleases (ZFNs) and transcription activator-like effector nucleases (TALENs) were created, but had shortcomings that rendered them unfeasible for use in live organisms. ${ }^{[4]}$ CRISPR promises to overcome these shortcomings by providing improved specificity, versatility, efficiency and accessibility. ${ }^{[5]}$

The CRISPR-Cas system is found in the adaptive bacterial immune system, and acts like molecular scissors. ${ }^{[4]} \mathrm{A}$ variant - CRISPR-Cas9 was applied successfully to the eukaryotic system by Doudna and Charpentier. ${ }^{[4]}$ Through this novel application of a simple bacterial genome-editing system to a eukaryotic cell, scientists realised the value of CRISPR-Cas variants in targeting, marking, editing, modifying and regulating the genome with greater ease, precision, efficiency and cost-effectiveness. ${ }^{[4]}$

\section{Agri-biotechnology}

In agriculture, CRISPR-Cas technology has the potential to improve crop quality and yield, and to introduce disease resistance ${ }^{[6]}-$ for example, genome-edited mushrooms that brown slower than other mushrooms, so increasing their shelf-life. ${ }^{[7]} \mathrm{GMO}$ s aimed to achieve the same goals, but required the introduction of foreign DNA, resulting in transgenes. A main point of contention with GMOs, then, was how to prevent the cross-pollination of genetically engineered plants with their relative plants in the wild. ${ }^{[1]}$ As such, regulations in the agricultural use of GMOs focused on preventing GMO release in the environment or market in ways that may impact wild types.

The Genetically Modified Organisms Act No. 15 of 1997 (GMO Act) outlines the requirements of an application to pursue GMOs. These requirements include scientifically based risk assessment, socioeconomic considerations, environmental impact and risk management measures. ${ }^{[8]}$ Other SA acts concerned with the 
mitigation of the environmental impact of GMOs include the National Environmental Management Act No. 107 of 1998, and the National Environmental Management Biodiversity Act No. 10 of 2004.

These statutes focus on GMOs, with the reason for stringently regulating GMOs, as seen in the Cartagena Protocol on Biosafety in 2003 , being that GMOs incorporate foreign DNA (transgenes), and the view advanced by some that the long-term effect on the environment and consumer health of transgenic organisms is unknown. However, genome editing does not require the incorporation of foreign DNA; it is a process of mutagenesis, not transgenesis. To clarify this point further, genetically edited organisms do not possess DNA that exists outside their gene pool, where gene pool refers to the genes present within a population of naturally interbreeding species. By this definition, gene editing is seen almost as an enhanced natural breeding process; that is, the DNA does not undergo any changes that normal reproductive processes cannot hypothetically achieve. This contrasts with gene modification, wherein DNA that does not naturally exist in the gene pool is added to achieve a certain trait. In this scenario, under conventional reproduction, these foreign genes would not typically enter the gene pool of the organism, and may confer traits that are atypical.

This is a pivotal fact to consider when answering the question that is being raised on whether these regulations on GMOs can be applied to genome-edited organisms. Some maintain that genome editing using CRISPR-Cas technology does not pose any greater threat than conventional breeding practices, as there is no introduction of foreign DNA into the organism. ${ }^{[9]}$ An example of this is the emerging policy in the USA that the Food and Drug Administration (FDA) does not regulate CRISPR-Cas technology end-products, such as genetically edited mushrooms ${ }^{[7]}$ and maize, ${ }^{[10]}$ as these do not contain DNA from plant pests such as viruses and bacteria, and are therefore not transgenic.

In Europe, the issue of whether genome-edited organisms would be regulated under current GMO legislation ${ }^{[1]}$ was brought to the European Court of Justice by a French agricultural union of smallscale farmers, in the case of Confédération Paysanne and Others v Premier Ministre and Ministre de I'Agriculture, de I'Agroalimentaire et de la Forêt C-528/16. The agricultural union argued that although mutagenesis is excluded from regulation, more modern methods of mutagenesis - genomic editing - are threatening the health of humans and the environment and should not be exempt from regulation. In 2016, the French government asked the European Court of Justice to provide clarity on the interpretation of the directive that regulated GMOs - essentially asking whether products of new methods of genome editing fell under the mutagenesis exemption. Before deciding a matter, the European Court of Justice can request an independent legal opinion from its panel of Advocates General. Such opinions are influential in the court's eventual decision, and are followed in about two-thirds of decisions. In this case, Adv. Gen. Michal Bobek was requested to provide an opinion, and he filed his opinion in January 2018. ${ }^{[12]} \mathrm{He}$ opines that products created using mutagenesis techniques fall under the ambit of the mutagenesis exemption, except in certain cases - such as where recombinant nucleic acid molecules are used, or where DNA that does not occur naturally is introduced. ${ }^{[12]}$ This means that genomic editing may, in many cases, fall outside the ambit of the European GMO regulatory framework. However, Adv. Gen. Bobek points out that individual member states still have the authority to enforce their own regulations of gene-edited products and processes..$^{[12]}$ The decision by the European Court of Justice, as of July 2018, is that genetically edited plants are subject to the same laws as GMOs.

In SA, the GMO Act's definition of a GMO is 'an organism the genes or genetic material of which has been modified in a way that does not occur naturally through mating or natural recombination or both. ${ }^{[8]}$ This definition clearly mirrors GMO definitions in the USA, European Union and elsewhere. As such, as evidenced by our discussion of foreign jurisdictions, it is conceivable that SA regulators and courts would view genome-edited organisms as in some instances falling within and in some instances falling outside this definition - depending on the exact nature of the genome editing that took place. However, lobby groups are already taking positions: the Africa Centre for Biodiversity calls for a strict ban on genome-edited techniques until the 'risks' of the products can be assessed. ${ }^{[13]}$ Though the report distinguishes between the method used to create GMOs and that of mutagenesis, the report raises two overall concerns that have been mentioned above: (i) mutagenesis as a technique cannot be disregarded in terms of safety and scientific validity; and (ii) the products of genome editing, though acquired by mutagenesis, may still be classified as GMOs. Lobbyists also argue that the use of recombinant DNA still introduces foreign DNA to the organism. The Africa Centre for Biodiversity expands on other existing concerns regarding the scientific feasibility of mutagenesis, claiming that around $70 \%$ of mutations lead to detrimental and not beneficial effects'.[13] However, such sweeping statements seem to ignore the evolution of mutagenesis techniques from those that caused widespread and unspecific mutations, such as irradiation, to that of CRISPR, which promises better specificity. Essentially, the Africa Centre for Biodiversity echoes the question raised by the international community - do current GMO regulations pertain to genome editing? Clearly, legal certainty is needed. If the question is answered similarly to the way it has been answered in the USA, and in the opinion of Adv. Gen. Bobek, SA may also consider genomeediting-specific legislation to address the regulatory gap.

\section{Gene editing in human somatic cells, germlines and embryos}

The current literature focuses largely on the scientific risks and benefits, and also the ethics, of altering somatic cells vis-à-vis germline and embryonic cells. ${ }^{[14]}$

\section{Somatic cells}

The main question raised in the alteration of somatic cells is whether scientists can accurately predict how editing a specific set of genes can affect the rest of the genome, and whether possible adverse effects and risks can be sufficiently understood. However, none of these questions can be fully answered without conducting extensive research. In 2016, the USA approved the first protocol for gene therapy that uses CRISPR-Cas technology. ${ }^{[5]}$ The first phase I clinical trial of using CRISPR-modified lymphocytes against lung cancer in humans is imminent in China. ${ }^{[5]}$

While the current safety of somatic-cell gene therapy is still being investigated, it is relatively uncontroversial ethically, and will be 
regulated as 'medicine' by the Medicines and Related Substances Act No. 101 of 1965. Not all uses of somatic-cell gene editing will necessarily be therapy - some conceivable uses of somatic cell gene editing can be viewed as non-therapeutic, or 'enhancement'. Although the therapy-enhancement distinction is popular among bioethicists to draw the line between acceptable and non-acceptable uses of genetic technology in humans, there is no consensus on this issue. In fact, there are examples in conventional medicine where medical technology is used for non-therapeutic purposes. Cosmetic surgery is perhaps the most apparent example. Other examples may include the use of concentration-enhancing psychiatric medicines that were intended to treat conditions such as ADHD by people who do not have ADHD or a similar condition. How one perceives enhancements will depend on how one interprets and balances norms such as autonomy and fairness.

\section{Germline and embryonic cells}

Germline and embryonic cells are a more contentious area of gene therapy. Germline cells develop into the reproductive cells of an individual, and alteration of these cells is heritable; that is, the alteration will be passed onto the offspring resulting from those reproductive cells. The key issue here is similar to that of somatic cells - that not all the risks to the rest of the genome are known - but now it also concerns the genomes of the offspring. Embryonic-cell gene editing shares this concern, but there is an added dimension of whether genome-edited embryos can be safely implanted in the uterus.

The United Nations Educational, Scientific and Cultural Organization (UNESCO) has taken a conservative stance, calling for a temporary ban on any use of germline gene editing ${ }^{[3]}$ The US National Academy of Sciences and the National Academy of Medicine released a report entitled Human Genome Editing: Science, Ethics, and Governance, in which a slightly more flexible approach is adopted that allows for clinical trials of germline gene editing if ten criteria are met. ${ }^{[15]}$ Internationally, according to a survey of 39 countries - including the UK, USA, China and SA - conducted by Araki and Ishii, ${ }^{[16]}$ there is dissidence regarding the ban on human germline editing. China does not have a legal ban, but rather a guideline-based ban on germline editing, ${ }^{[16]}$ and takes the Confucian view that because the status of human beings is only acquired upon birth, and not before, the use of embryos for research is permissible. ${ }^{[17]}$

It is therefore unsurprising that the first study reporting CRISPR genetic editing of human embryos was conducted by a research team in China. Liang et al. ${ }^{[18]}$ used non-viable embryos with a triploid set of chromosomes to investigate CRISPR-Cas9-mediated gene editing ${ }^{[18]}$ The tool effectively cleaved the desired HBB $\beta$-globin gene; however, the efficiency of the gene repair after cleavage was low. Furthermore, there was also off-target gene cleavage and mutations. While this is not scientifically feasible for progression to clinical trials, it is a critical first step in identifying the challenges when using CRISPR technology in embryos. ${ }^{[18]}$ Indeed, this research was the focus area of the 2015 International Summit on Human Gene Editing held in Washington DC by the US National Academy of Sciences, the US National Academy of Medicine, the Royal Society, and the Chinese Academy of Sciences. ${ }^{[17]}$ The final position was that the research should not be condemned, and though there was disagreement in the scientific community about the extent of applying gene editing to embryos, some countries have recognised the value of allowing research in germline editing. ${ }^{[17]}$

Though current UK legislation, namely the Human Embryology and Fertilisation Act, prohibits the implantation of genetically modified embryos, ${ }^{[1]}$ there is no legal ban on germline editing ${ }^{[16]}$ and research into this area of genetic editing of the germline and embryos may continue as long as it is licensed by the Human Fertilisation and Embryology Authority. ${ }^{[6]}$ The USA has taken a more cautious approach, with the FDA and National Institutes of Health placing a temporary moratorium on germline gene editing until sufficient risk and safety assessments have been conducted. ${ }^{[16]}$ Other countries such as Sweden and Canada have banned germline editing, maintaining that this practice should be banned because it may affect the development of the embryo. As Araki and Ishii ${ }^{[16]}$ point out, whether this reasoning will still stand if gene correction leads to normal development of the embryo, is uncertain.

SA's regulatory regime regarding germline genome editing is perceived as ambiguous. ${ }^{[16]}$ This is not surprising, as the National Health Act No. 61 of 2003 bans 'reproductive cloning' of humans, and appears to define 'reproductive cloning' so broadly as to possibly include any germline genome editing. Given this strange definition, legal certainty is compromised.

\section{Patenting CRISPR technology}

Despite the ongoing patent litigation, the University of California and the Broad Institute, the Massachusetts Institute of Technology (MIT) and Harvard University have all proceeded to grant broad exclusive licences to use their respective CRISPR technologies commercially; ${ }^{[19]}$ the Broad Institute, MIT and Harvard state that academic and nonprofit use shall not require licences. ${ }^{[20]}$ For example, the Broad Institute employs an 'inclusive innovation model' wherein the surrogate company Editas is a primary licensee of exclusive licences, but after an initial period, other companies may be granted licences for the CRISPR technology. ${ }^{[20]}$ Sherkow ${ }^{[19]}$ voices the concern that this may prevent other smaller companies from innovating using CRISPR-Cas9 technology, and suggests that co-operative patent licensing should be encouraged, as opposed to exclusivity and patenting thickets. The University of California and the Broad Institute have announced that they are working on a patent pool and other mechanisms to enhance access to CRISPR technology, but this requires foundational patent owners ${ }^{[21]}$ which include the company Šikšnys and other agricultural companies such as Monsanto, DuPont Pioneer, Caribou Biosciences, and ERS Genomics. ${ }^{[19]}$

Apart from the issue of access to technology, there is also the issue of whether down-the-line inventions using CRISPR technology would even be patentable. Sherkow ${ }^{[19]}$ questions whether there is any non-obvious application of CRISPR-Cas9, with obvious negative consequences for patentability. ${ }^{[19]}$ The process itself has already been patented, and it may be possible that future patents will include the use of the technique in claims to the genome-edited end products. However, what is debatable is: (i) whether these gene-edited end products are patentable subject matter, as they may be regarded as products of nature, ${ }^{[22]}$ a debate predating genome editing; (ii) whether these gene-edited end products, if they are homologous to corrected genes present in a normal healthy population, fulfil the novelty criterion of patentability; ${ }^{[22]}$ and (iii) whether the production 
of the edited genes introduces a non-obvious step that is required for patent eligibility. ${ }^{[22]}$

A search on 'CRISPR' through the SA patent database shows that there is one CRISPR patent granted in SA - to a Swiss company CRISPR Therapeutics. Another patent application that was filed by MIT, the Broad Institute and the University of lowa has been accepted, but is not yet granted. A further 12 patent applications by various institutes or companies in the USA are pending in the SA Patent Office (SAPO). It should be remembered that SAPO does not examine the substance of patent claims, as the USA and the EU do - however, SAPO aims to move in this direction. As such, the fact that a certain patent has been granted in SA does not necessarily reflect on the legal tenability of the patent's claims. In the current system, this can only be tested in court. Given the fact that SAPO intends to move towards an examining system, it would assist with legal certainty if SAPO could articulate a position regarding the patentability of CRISPR technology and genome-edited end products in SA.

\section{Regulating the consumer market}

Another issue involving the protection and promotion of human health is the marketing and labelling of genome-edited foods. ${ }^{[1]}$ In SA, the Consumer Protection Act No. 68 of 2008, which defines GMOs as defined in section 1 of the GMO Act, provides that GMO content of certain categories be noted on food labels. ${ }^{[23]}$ If gene-edited foods are not considered as GMOs, this may avoid the labelling regime, and may also avoid possible rejections by some sectors of the consumer public. ${ }^{[24]}$

\section{Conclusion}

CRISPR technology affects a whole range of ethical and legal fronts. What should be clear from our discussion is that SA faces challenges on all of these fronts, and requires greater legal clarity regarding the regulation of this new technology. By identifying these fronts, we hope to stimulate academic analysis in the SA context. In summary, such academic analysis should aim at the following most pertinent areas where legal clarity is most required: (1) Whether CRISPR-Cas9 falls within the ambit of the GMO Act's definition of a GMO, and if not, whether there are convincing public policy reasons for amending the Act to include gene-edited foods within its regulatory ambit. (2a) Whether there is an ethical limit to the application of genome editing in humans, and if so, how such limit should be regulated in law. (2b) Whether human germline editing qua specific kind of genome editing in humans should be legally banned, regulated, or unregulated, and if regulated, the conditions and oversight mechanisms should be given good thought. (3) In the context of patenting, where there are overlapping claims, which should prevail, and given the breadth of the prevailing claims, whether there is space for patenting genome-edited end products in SA. (4) Lastly, having regard to the answer to point (1) above, whether gene-edited foods should require labelling similar to the current GMO labelling. Not one of these areas has an easy or obvious answer - each one calls for in-depth academic analysis in the SA context. Science itself cannot tell us what to do. We introduce norms to facts.
Acknowledgements. None

Author contributions. Equal contributions.

Conflicts of interest. None.

Funding. None.

1. Shepperd S. The revolutionary gene editing technology: CRISPR-Cas9. eHealth Law Policy 2016;3(6):11-13.

2. Chen JS, Ma E, Harrington LB, et al. CRISPR-Cas12a target binding unleashes indiscriminate single-stranded DNase activity. Science 2018;360(6387):436-439. https://doi.org/10.1126/science.aar6245

3. Howard HC, van El CG, Forzano F, et al. One small edit for humans, one giant edit for humankind? Points and questions to consider for a responsible way forward for gene editing in humans. Eur J Hum Genet 2018;26(1):1-11. https:// doi.org/10.1038/s41431-017-0024-z

4. Doudna JA, Charpentier E. The new frontier of genome engineering with CRISPRCas9. Science 2014;346(6213):1258096. https://doi.org/10.1126/science.1258096

5. De Lecuona I, Casado M, Marfany G, Lopez Baroni M, Escarrabill M. Gene editing in humans: Towards a global and inclusive debate for responsible research. Yale J Biol Med 2017;90(4):673-681.

6. Rodriguez E. Ethical issues in genome editing using CRISPR/Cas9 system. J Clin Res Bioeth 2016;7:266. https://doi.org/10.4172/2155-9627.1000266

7. Waltz E. Gene-edited CRISPR mushroom escapes US regulation. Nature 2016:532(7599):293. https://doi.org/10.1038/nature.2016.19754

8. South Africa. Genetically Modified Organisms Act No. 15 of 1997.

9. Gao C. The future of CRISPR technologies in agriculture. Nat Rev Mol Cell Biol 2018;19(5):275-276. https://doi.org/10.1038/nrm.2018.2

10. Ishii T, Araki M. A future scenario of the global regulatory landscape regarding genome-edited crops. GM Crops Food 2017;8(1):44-56. https://doi.org/10.1080 /21645698.2016.1261787

11. European Union. Regulation (EC) No. $1829 / 2003$ of the European Parliament and of the Council on genetically modified food and feed. 2003.

12. Bobek M. Opinion of Advocate General Bobek on Case C-528/16. 2018. http:// curia.europa.eu/juris/document/document.jsf?text=\&docid=198532\&pagelnde $\mathrm{X}=0$ \&doclang $=\mathrm{EN} \&$ mode $=\mathrm{req} \& \mathrm{dir}=\& \mathrm{occ}=$ first\&part $=1$ (accessed 10 May 2018).

13. Africa Centre for Biodiversity. Biosafety Risks of Genome Editing Techniques in Plant Breeding. 2017. https://bangmosnowdotcom.files.wordpress.com/2017/02/ biosafety-genome-editing-pb.pdf (accessed 18 May 2018).

14. Wolpe PR, Rommelfanger KS, Drafting and Reviewing Delegates of the BEINGS Working Groups. Ethical principles for the use of human cellular biotechnologies. Nat Biotechnol 2017;35(11):1050-1058. https://doi.org/10.1038/nbt.4007

15. National Academies of Science and National Academy of Medicine. Human Genome Editing:Science, Ethics and Governance. 2017. http://nationalacademies. org/cs/groups/genesite/documents/webpage/gene_177255.pdf (accessed 16 May 2018).

16. Araki M, Ishii T. International regulatory landscape and integration of corrective genome editing into in vitro fertilization. Reprod Biol Endocrinol 2014;12(1):108 https://doi.org/10.1186/1477-7827-12-108

17. Reardon S. Global summit reveals divergent views on human gene editing Nature 2015;528(7581):173. https://doi.org/10.1038/528173a

18. Liang $\mathrm{P}, \mathrm{Xu} \mathrm{Y}$, Zhang $\mathrm{X}$, et al. CRISPR/Cas9-mediated gene editing in human tripronuclear zygotes. Protein Cell 2015;6(5):363-372. https://doi.org/10.1007/ s13238-015-0153-5

19. Sherkow JS. The CRISPR patent landscape: Past, present, and future. CRISPR J 2018:1(1):5-9. https://doi.org/10.1089/crispr.2017.0013

20. Broad Institute. Information about licensing CRISPR genome editing systems. 2018. https://www.broadinstitute.org/partnerships/office-strategic-alliances-andpartnering/information-about-licensing-crispr-genome-edi (accessed 12 May 2018).

21. Holtz B. CRISPR/Cas9 technology intellectual property \& economic issues. ARRIGE Kick-off Meeting; 2018 Mar 25. https://arrige.org/ARRIGE_patents_Holtz.pdf (accessed 12 May 2018)

22. Ku D. The patentability of the Crispr-Cas9 genome editing tool. Chicago-Kent Journal of Intellectual Property 2017;16(2):408-439.

23. South Africa. Consumer Protection Act No. 68 of 2008. Section 7, 293. 2008.

24. Abbott A. European court suggests relaxed gene-editing rules. Nature 19 January 2018. https://doi.org/10.1038/d41586-018-01013-525

25. Smalley E. FDA warns public of dangers of DIY gene therapy. Nat Biotechnol 2018;36(2):119-20. https://doi.org/10.1038/nbt0218-119 